\title{
Comparisons between EUV/IMAGE observations and numerical simulations of the plasmapause formation
}

\author{
V. Pierrard ${ }^{1}$ and J. Cabrera ${ }^{2}$ \\ ${ }^{1}$ Belgian Institute for Space Aeronomy, Brussels, Belgium \\ ${ }^{2}$ Center for Space Radiations, UCL, 1348 Louvain-La-Neuve, Belgium \\ Received: 8 March 2005 - Revised: 6 July 2005 - Accepted: 3 August 2005 - Published: 14 October 2005
}

\begin{abstract}
Simulations of plasmapause formation described in Pierrard and Lemaire (2004) predict the shape and equatorial distance of the plasmapause as a function of the geomagnetic activity index $K_{p}$. The equatorial positions predicted by this model are compared with the observations of EUV/IMAGE during the geomagnetic storm of 24 May 2000, substorm events of 10 June 2001 and 25 June 2000, and also during a prolonged quiet period (2 May 2001) when the plasmasphere was very extended. The formation of structures, like plumes and shoulders observed during periods of high geomagnetic activity, is quite well reproduced by the simulations. These structures are directly related to specific time sequences of $K_{p}$ variations. The radial distances of the plasmapause are also reproduced, on average, by the model.
\end{abstract}

Keywords. Magnetospheric physics (Plasmasphere; Storms and substorms; Solar wind magnetosphere interactions)

\section{Introduction}

The plasmasphere constitutes the extension of the ionosphere at high altitude: cold plasma from the ionosphere moves along magnetic field lines and populates flux tubes forming the plasmasphere. The outer surface in the plasmasphere is often characterized by a sharp decrease in the plasma density, called the plasmapause. Whistlers and in-situ satellite observations have revealed that the equatorial position of the plasmapause varies from $2.5 R_{E}$ to $7 R_{E}$ and depends, on average, on the level of geomagnetic activity (Carpenter and Anderson, 1992; Moldwin et al., 2002).

The first global comprehensive images of the Earth's plasmasphere were provided by the EUV (Extreme UltraViolet) instrument on board the IMAGE spacecraft launched in March 2000 (Burch et al., 2001). With these images, the large-scale dynamics of the plasmasphere was revealed. Irregular structures at the plasmapause, like shoulders, plumes

Correspondence to: V. Pierrard

(viviane.pierrard@aeronomie.be) and channels were identified and analyzed (Sandel et al., 2003).

These new observations give an exceptional opportunity to check the mechanisms proposed for the formation of the plasmapause. It is well admitted that the configuration of cold plasma in the plasmasphere depends on the electric field. This electric field is formed by the interplay of the co-rotation electric field, which dominates near Earth, and the convection electric field, generated by the interaction of the solar wind with the magnetosphere. When the geomagnetic activity level increases, the convection electric field increases and the region where co-rotation is enforced shrinks. The outer streamlines are then depleted. On the contrary, when the convection electric field diminishes, the co-rotation region expands to include some depleted flux tubes, which can then refill by evaporation from the ionosphere and by other possible mechanisms.

In the first theoretical formulation of the plasmasphere, the plasmapause coincided to the last closed equipotential of the electric field after prolonged periods of constant $K_{p}$ values. Unfortunately, the magnetospheric electric field is never stationary long enough for such an ideal plasmapause frontier to form. On the contrary, all observations since 1963 indicate that the formation of a sharp plasmapause occurs during magnetic substorm events, when the magnetosphere becomes suddenly disturbed.

Another mechanism to explain the plasmapause formation has been proposed (Lemaire, 1974; 1985; 2000): the cold plasma distribution becomes convectively unstable in the outermost region of the plasmasphere when the convection electric field is suddenly enhanced. The centrifugal force drives the plasma upwards and then produces a sharp density gradient along the magnetic field lines tangent to the surface where the parallel force is zero (Zero Parallel Force Surface: ZPFS). This mechanism is described in detail in Lemaire and Gringauz (1998). According to this mechanism, the plasmapause is determined by a convective instability in the postmidnight sector, where the convection velocity is maximum. After an increase in the level of magnetic activity, i.e. an 
increase in the $K_{p}$ value, a new plasmapause is formed closer to the Earth than the last closed equipotential of the electric field.

When $K_{p}$ increases, the plasmasphere is peeled off in the post-midnight LT sector. Due to the depletion of the outer flux tubes, the plasmapause is closer to the Earth in this LT sector. A few hours later, a plume is often generated in the afternoon LT sector due to differential rotation. When $K_{p}$ decreases, the ZPFS shifts to larger radial distances. The outer flux tubes beyond the innermost vestigial plasmapause refill until a level of saturation is achieved. If the decrease in $K_{p}$ is abrupt, a shoulder like those observed by EUV can develop.

Other explanations have been proposed for the formation of plumes (Grebowsky, 1970) and shoulders (Goldstein et al., 2002). They are based on variations of the convection electric field with parameters and boundary conditions appropriately adjusted. By such adjustments, these authors were able to fit the results of their models with a number of observed plasmapause positions.

The simulations presented in the present paper use a given $K_{p}$-dependent magnetospheric electric field model without any adjustment. This electric field is the E5D model determined from dynamical proton and electron spectra measured on board the geostationary satellites ATS-5 and 6 (McIlwain, 1986). The empirical electric field E5D is fully determined by the value of $K_{p}$. These simulations developed at IASB (Pierrard and Lemaire, 2004) are based on the mechanism of instability for the plasmapause formation and thus depend on the values of $K_{p}$.

The results of the simulations based on the E5D electric field have been compared with CLUSTER data (Dandouras et al., 2004). The fit between predictions of this model and the CLUSTER observations was quite satisfactory. In the present paper, we compare the prediction of this same model with the global EUV/IMAGE observations of the plasmasphere. The chosen case studies correspond to substorm events, magnetic storms and prolonged quiet periods.

\section{Methodology}

The physical mechanisms and the numerical method on which the following simulations are based, are explained, respectively, in Lemaire and Gringauz (1998) and Pierrard and Lemaire (2004). The $K_{p}$ index during the period of time is the only input of the time-dependent model. $K_{p}$ determines uniquely the E5D convection electric field distribution. The post-midnight sector is the region where the convection electric field intensity is the largest and where the plasmapause peeling off is expected to take place according to this model.

The plasmapause is formed at the equatorial distance of the deepest penetration of the ZPFS: this is located around 02:00 LT for the E5D model. At subsequent LT, the plasmapause position is determined by the earlier values of $K_{p}$, resulting from the changing electric field distribution and the $(\boldsymbol{E} \times \boldsymbol{B}) / \mathrm{B}^{2}$ drift motion of the plasmapause.
The model predictions are compared with EUV observations from IMAGE. These observations are intensity maps of the $30.4 \mathrm{~nm}$ emissions of Helium ions integrated along the line of sight. They are projected in the geomagnetic equatorial plane in the SM reference system with the program XForm (ftp://euv.lpl.arizona.edu/pub/bavaro/unsupported/), in order to have the same view over the pole as in the simulations. The plasmapause is assumed to be the sharp edge where the brightness of $30.4 \mathrm{~nm} \mathrm{He}^{+}$emissions drops drastically. To better visualize the plasmapause, we draw a red line corresponding to $40 \%$ of the maximum intensity of the image, where the intensity is the logarithm of the luminosity.

\section{During a magnetic substorm}

Periods of time following an increase in $K_{p}$ or a magnetic substorm event are particularly interesting to study because they generally show the development of a so-called plume in the plasmasphere. This kind of plume is often observed in IMAGE observations and follows even moderate increases of $K_{p}$ (above $\left.3^{+}-4\right)$.

\section{$3.19-10$ June 2001}

Let us show the example of 9-10 June 2001, for which EUV observations were presented by Spasojevic et al. (2003). The upper panel of Fig. 1a shows $B_{z}$, the $z$ component of the interplanetary magnetic field, the $D_{s t}$ index and the geomagnetic activity index $K_{p}$, observed from 8 June to 10 June 2001. During this period of 3 days, $K_{p}$ gradually increases up to $5^{+}$and then decreases. Note that during this geomagnetic substorm, the interplanetary magnetic field turns southward during more than $5 \mathrm{~h}$.

The lower panel of Fig. 1a shows the result of the simulation on 9 June 2001 at 08:00 UT. $K_{p}$ is then observed to be equal to $2^{+}$. Due to the rather low value of $K_{p}$, the model predicts a plasmapause quite far from the Earth, around $4 R_{E}$. Because $K_{p}$ was low and almost constant during the previous $24 \mathrm{~h}$, the plasmapause is quasi-circular. The circles on the figure correspond to a radial distance of 1,2, 4 and 6 .

The result of the simulation is compared with the plasmasphere observed by EUV on 9 June 2001 at 08:00 UT, illustrated in Fig. 1b. The plasmasphere is viewed from a point of view about the North Pole and projected in the geomagnetic equatorial plane. The circles on the figure correspond to a radial distance of 1, 2, 4, 6 and 8 Earth radii. One can see that the plasmapause is indeed quite circular and located near $\mathrm{R}=4 R_{E}$.

Figure 2a illustrates the plasmapause obtained at 16:00 UT, thus $8 \mathrm{~h}$ later. $K_{p}$ has slightly increased, so that the plasmasphere is eroded in the post-midnight sector above the ZPFS, due to plasma instability. The ZPF surface, illustrated by the black line in the post-dusk MLT sector, corresponds to the equatorial cross section, where the effective gravitational acceleration has a zero component parallel to the dipolar magnetic field lines. This surface crosses the 


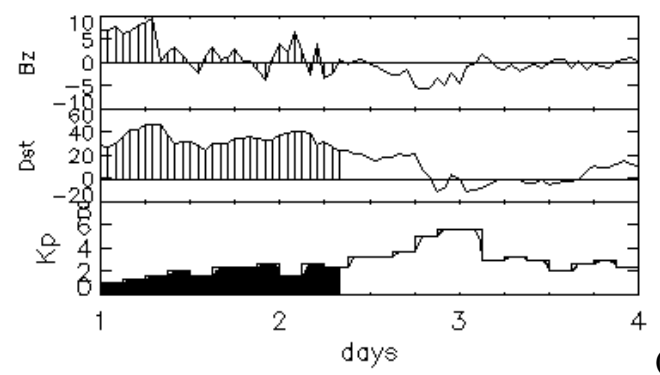

(a)

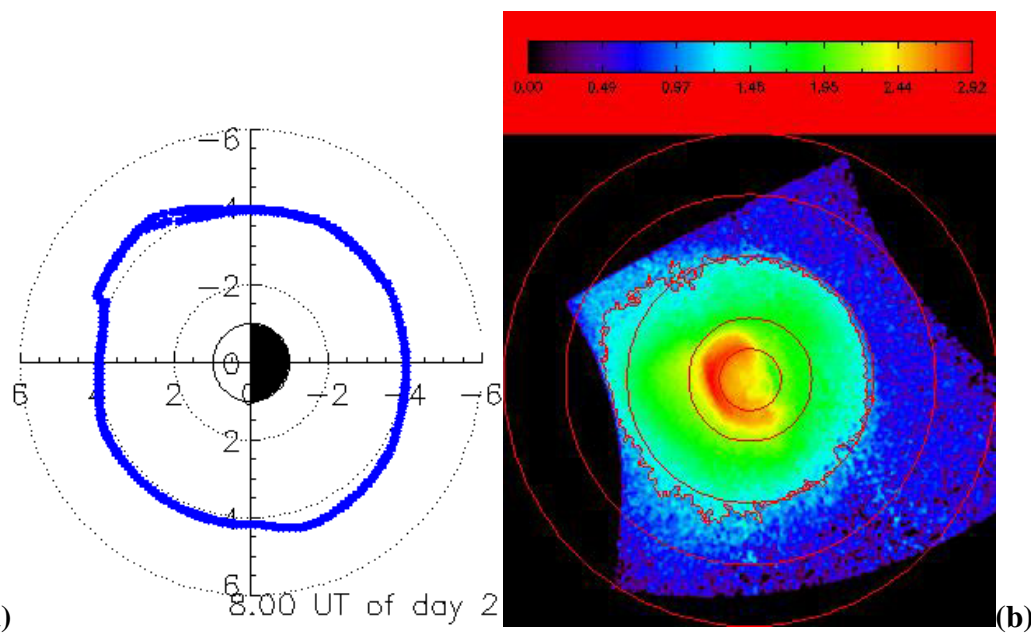

Fig. 1. (a) Result of the simulation based on the instability mechanism, the E5D model and the value of $K_{p}$ on 9 June $2001,08: 00$. The plasmapause in the geomagnetic equatorial plane corresponds to the blue line. The indexes $B_{z}, D_{s t}$ and $K_{p}$, observed during the previous and following days, are also displayed. The dotted circles correspond to L=1, 2, 4 and 6. (b) EUV observations on 9 June 2001 at 08:00 UT, projected in the geomagnetic equatorial plane. The red line corresponds to $40 \%$ of the maximum intensity of the image and permits one to visualize the plasmapause. The red circles correspond to $L=1,2,4,6$ and 8 .
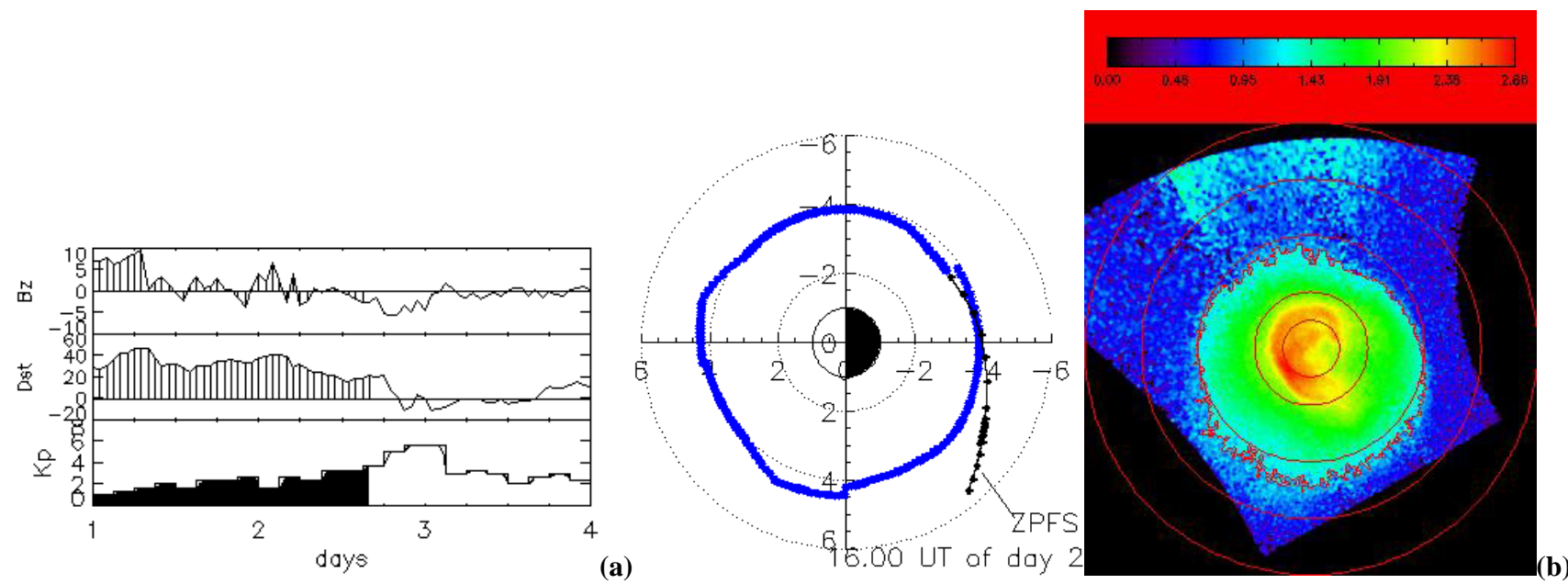

Fig. 2. Same as Fig. 1 for 9 June 2001, 16:00 UT ((a), simulation) and 16:04 UT ((b), EUV observation). The black line on the left panel corresponds to the Zero Parallel Force Surface.

plasmasphere only in the post-midnight sector and only when $K_{p}$ increases. This is why the plasmasphere is peeled off in this sector during geomagnetic substorms in the simulations. On Fig. 2b, one can see that the plasmasphere observed by IMAGE at 16:04 is indeed eroded in the post-midnight sector and that the plasmapause is formed closer to the Earth in this sector.

Figure 3a illustrates the plasmapause obtained at 23:00 UT (7 h later), when $K_{p}$ becomes maximum $\left(5^{+}\right)$. Again, the plasmasphere is peeled off in the post-midnight region due the increase in convection velocity, above the ZPFS illustrated by the black curve. Since the plasmasphere has rotated, the plasmapause is also close to the Earth in the morn- ing sector. Small structures that will form the plumes begin to appear at the plasmapause in the simulation. The EUV IMAGE observation in Fig. $3 \mathrm{~b}$ confirms that the plasmapause forms indeed closer to the Earth in the post-midnight region. But there are substantial differences between the two figures. Whereas the minimum radial expansion is at 02:00 03:00 LT in the model, it is at 07:00-08:00 LT in the IMAGE view. The small bump obtained in the simulation in the pre-noon sector is not observed by IMAGE. This bump is, in fact, due to the peeling off process considered in the simulation. Combined with the differential rotation, it leads to the development later on of a plume that becomes well visible in the afternoon sector in Figs. 4 a, b and c, corresponding, 

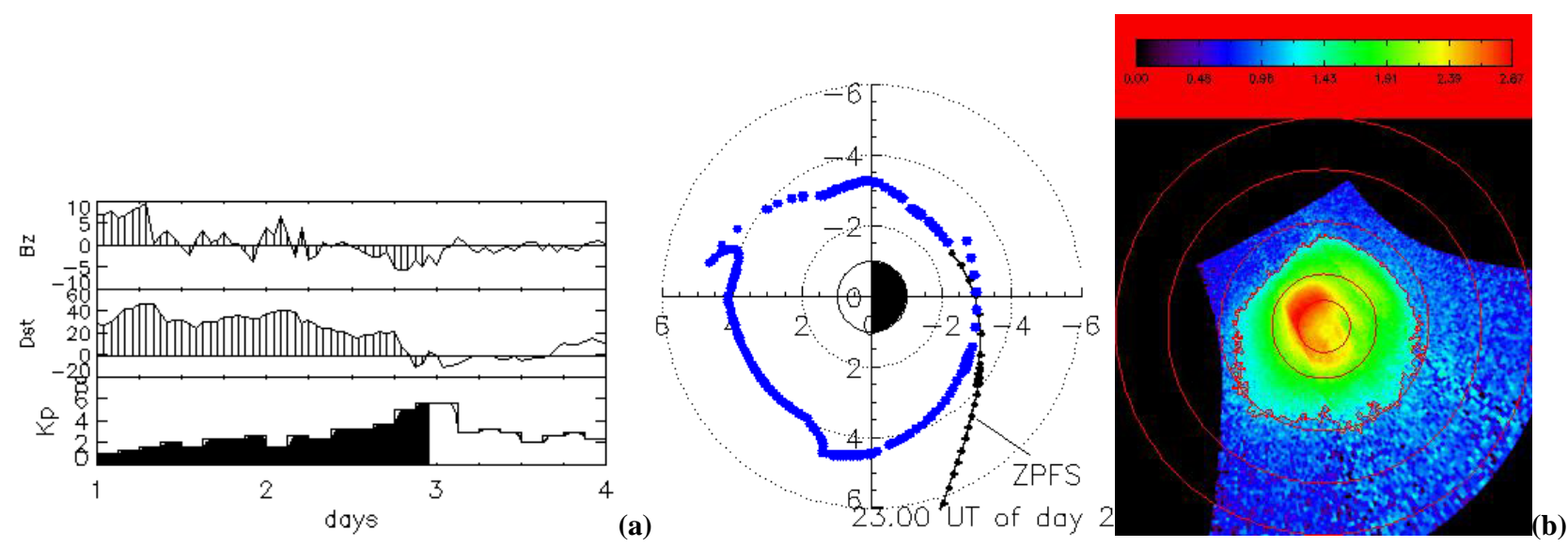

Fig. 3. Same as Fig. 1 for 9 June 2001, 23:00 ((a), simulation) and 23:04 UT ((b), EUV observation).

respectively, to 10 June 2001 at 01:00, 03:00, and 05:00 UT. The plumes in the simulations are shown by the blue dots that extend to large radial distances. The dots correspond to plasma elements located at the plasmapause.

Two different plumes (referred as "Plume 1" and "Plume 2 ") developed in the afternoon and dusk sector during this period of time. In the simulations, plumes develop after plasmaspheric erosion due to differential rotation, i.e. because the plasma convects more slowly around the Earth at large radial distance than closer to the Earth. Several plumes can sometimes develop during a period of time, corresponding to a $K_{p}$ increase. Such a plasmasphere with two different plumes has sometimes been observed by EUV.

Moreover, the sudden decrease in $K_{p}$ at 03:00 UT leads to the formation of a shoulder in the dawn sector, as seen on Fig. 4c. Indeed, when $K_{p}$ decreases, the plasmapause should extend further away from the Earth, since the ZPFS has then shifted to larger radial distances. Since the refilling process of the flux tubes is slower than the peeling off process and can sometimes take several days, we also draw the "vestigial plasmapause", which is then closer to the Earth in the dawn LT sector and appears after a $K_{p}$ decrease.

Unfortunately, it is not possible to compare the results of the simulation presented in Fig. 4 with EUV observations, since the IMAGE satellite is too close to the Earth to provide any global view of the plasmasphere during this period of time. The EUV observations are regularly interrupted along the orbit of the satellite, so that it is not easy to follow continuously the formation of plumes and their evolution with time. The observations have generally a typical duration of $7 \mathrm{~h}$ (with a time resolution of $10 \mathrm{~min}$ ) out of each 14 -h orbit. Just after an interruption, the satellite is still far from its apogee, so that the observations are not of very good quality, as is the observation on 10 June 2001 at 05:00 UT and this is why it is not shown in the present paper. But it is interesting to note that Plume 2 and the shoulder are already present at that time in the observations. The external edge of the plume is quite irregular at that time, so that Plume 1 can be roughly distinguished.
In Fig. 5a, we show the results of the simulation on 10 June 2001 at 07:00 UT. Plume 1 has become so thin that it has almost disappeared. The blue dots visible in the dusk and midnight sectors are the remains of this Plume 1. Plume 2 is well visible in the dusk LT sector. The shoulder has rotated.

Figure 5b shows the EUV/IMAGE observation at 07:00 UT. Plume 2 is indeed observed in the same LT sector as predicted by our simulations. The presence of a thin Plume 1 is not clear, but not excluded. The shoulder is clearly shown by the observations, but there is a slight delay in MLT compared to the model prediction, as if $K_{p}$ had in fact decreased earlier than in the measurements. Note that $K_{p}$ is a three-hour index, and leads to a $\pm 03: 00$ UT or \pm 3 MLT indetermination.

The presence of a well-structured shoulder at that time suggests another mechanism than the slow refilling of the plasma flux tubes, since the evaporation process from the ionosphere is generally quite inefficient in night-dawn LT sectors. As a complementary mechanism, interchange can also contribute to the shoulder formation: dips and blobs, trapped below the new ZPFS, rearrange so that the material internal to the new plasmapause boundary contributes to the shoulder shaped feature of the outer plasmasphere. Small-scale plasma density structures were indeed observed by CLUSTER mainly in the dusk sector during or after geomagnetic substorms (Darrouzet et al., 2004). These smallscale density structures, explained with the plasma instability theory (Lemaire, 1974), are not clearly seen at the same time in the corresponding EUV images, since the EUV instrument cannot resolve spatial structures with a size smaller than $0.1 R_{E}$ (Darrouzet at al., 2004). Moreover, small-scale density structures are difficult to detect by EUV, due to the line of sight integration and to the average lower threshold of the EUV corresponding to 40 electrons $/ \mathrm{cm}^{3}$ (Goldstein et al., 2004).

A few detached plasma elements were observed in situ in the post-midnight sector by Chappell et al. (1971), but at least some of these large-scale structures can correspond to corotating plumes. More recently, the EUV scanner on board 

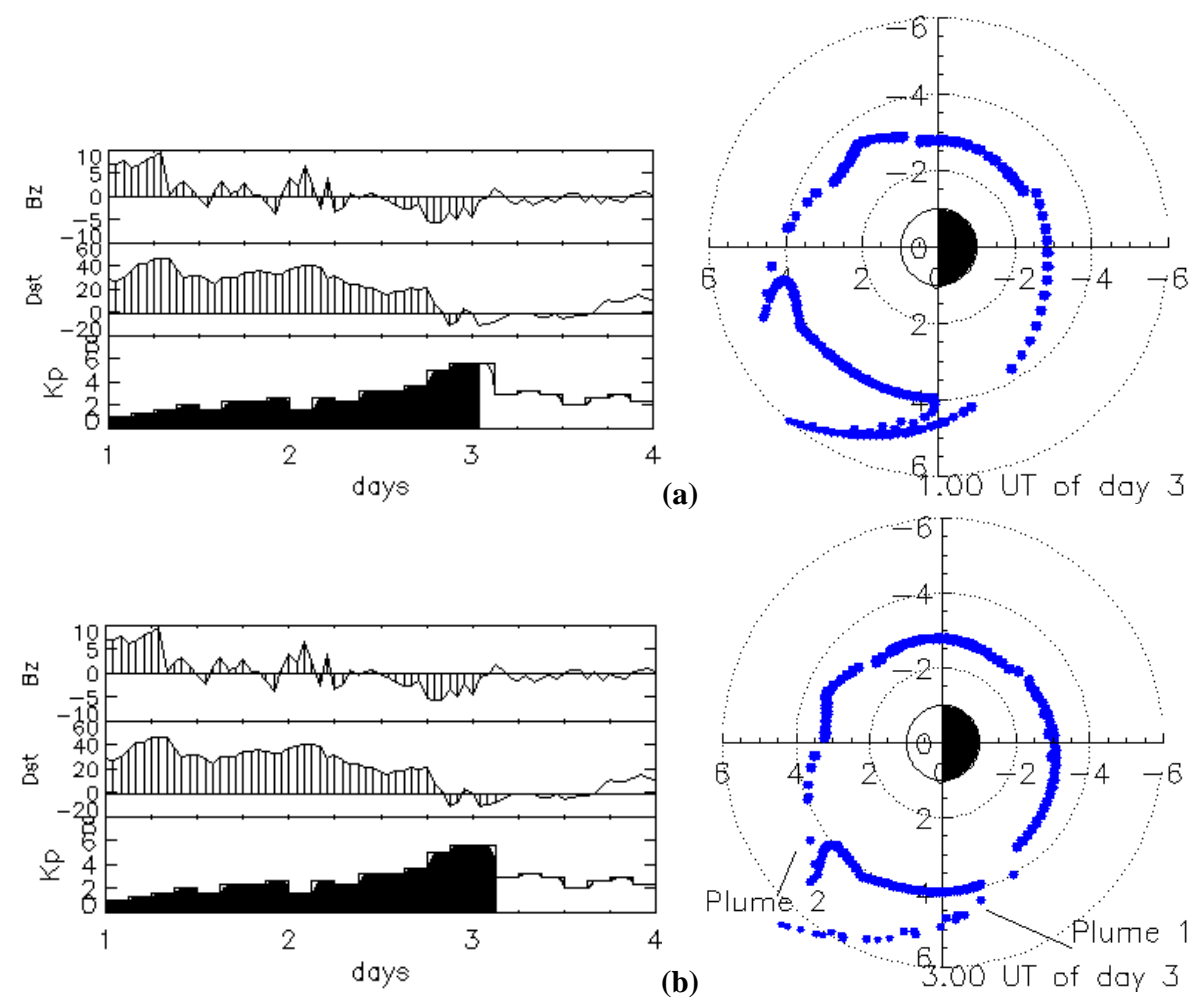

(b)

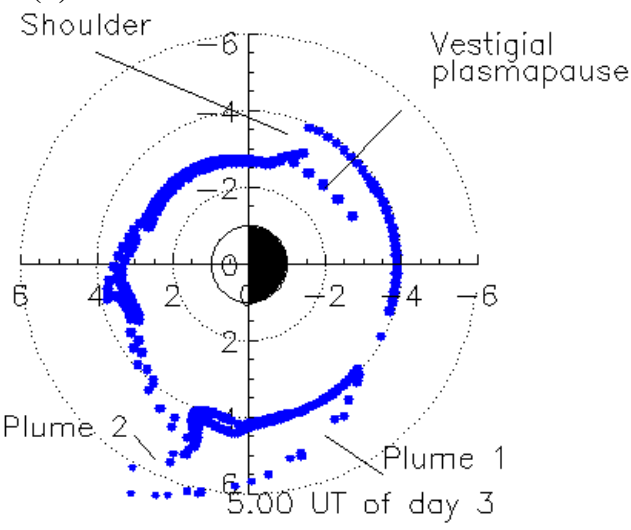

Fig. 4. Simulation of the development of plumes on 10 June 2001 at 01:00 UT (a), 03:00 UT (b) and 05:00 UT (c). IMAGE is too close to the Earth to provide a global view of the plasmasphere during this period of time.

Planet-B revealed that there is a significant amount of plasmaspheric ions escaping from the duskside outer plasmasphere toward the magnetosphere (Yoshikawa et al., 2000).

Note that the shoulder could also be due to an expansion of the plasmaspheric plasma up to the new plasmapause after the sharp decrease in $K_{p}$. In this case, the number density of the particles inside the inner plasmasphere should be slightly lower than before the formation of the shoulder. The EUV observations of Figs. $6 \mathrm{~b}$ and $7 \mathrm{~b}$ show that the shoulder continues to expand outwards even after 07:00 UT. More studies cases would be useful to determine the mechanisms involved in the formation of shoulders.

Later on, $K_{p}$ remains almost constant and small $\left(<2^{+}\right)$. The simulation shows how the plume and the shoulder ro- tate with the Earth and evolve. This can be seen in Figs. 6 a and $7 \mathrm{a}$, corresponding, respectively, to 10 June 2001, 10:00 and 12:00 UT. EUV observations at 09:57 UT in Fig. 6b and 12:00 in Fig. 7b show that the shoulder moves slowly outward. The clearly identifiable plume becomes thinner with time. The rotation seems quite slower in the dusk than in other LT sectors, so that the plume wraps and becomes captured. This process creates a density depletion that is mostly extended in azimuth, as shown in Fig. $7 b$.

In all EUV figures corresponding to the present case study, we use the threshold 0.40 of the maximum intensity to draw the limit of the plasmasphere. A lower threshold would give a red line a little bit further from the Earth, where the thin plasma depletion shown in Fig. $7 \mathrm{~b}$ is less indented. The 

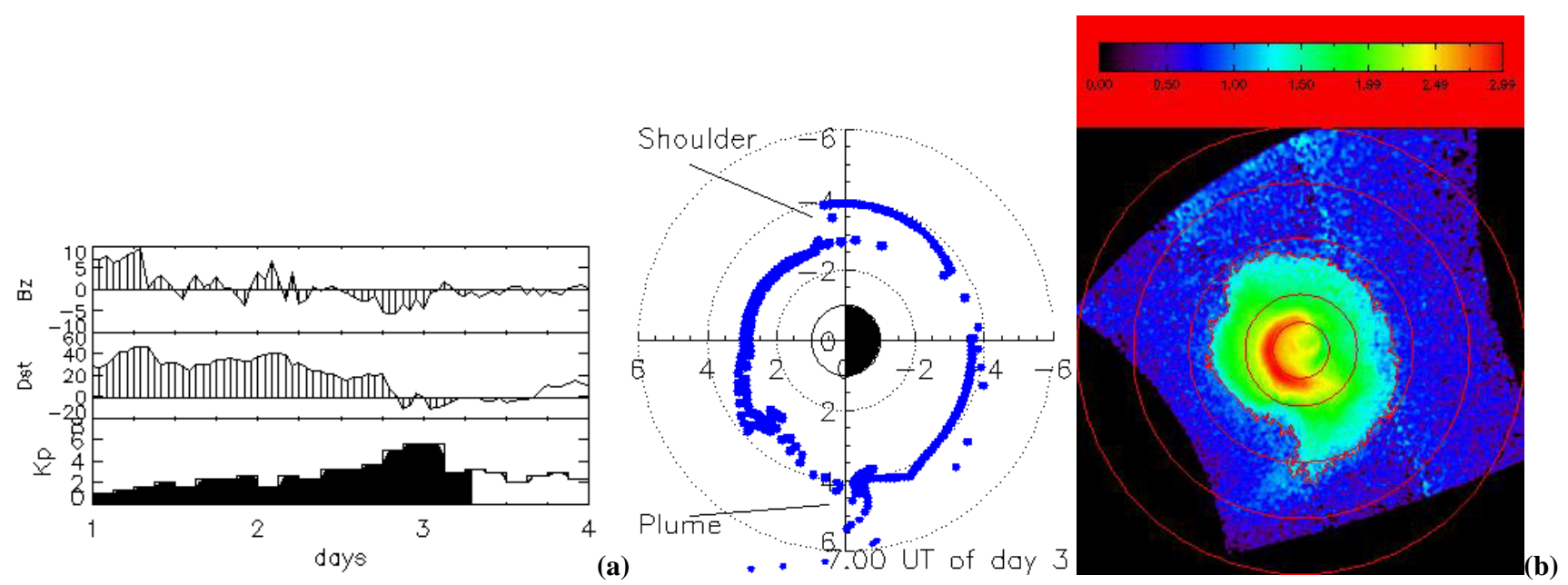

Fig. 5. Same as Fig. 1 for 10 June 2001, 07:00 UT ((a), simulation) and 07:00 UT ((b), EUV observation).
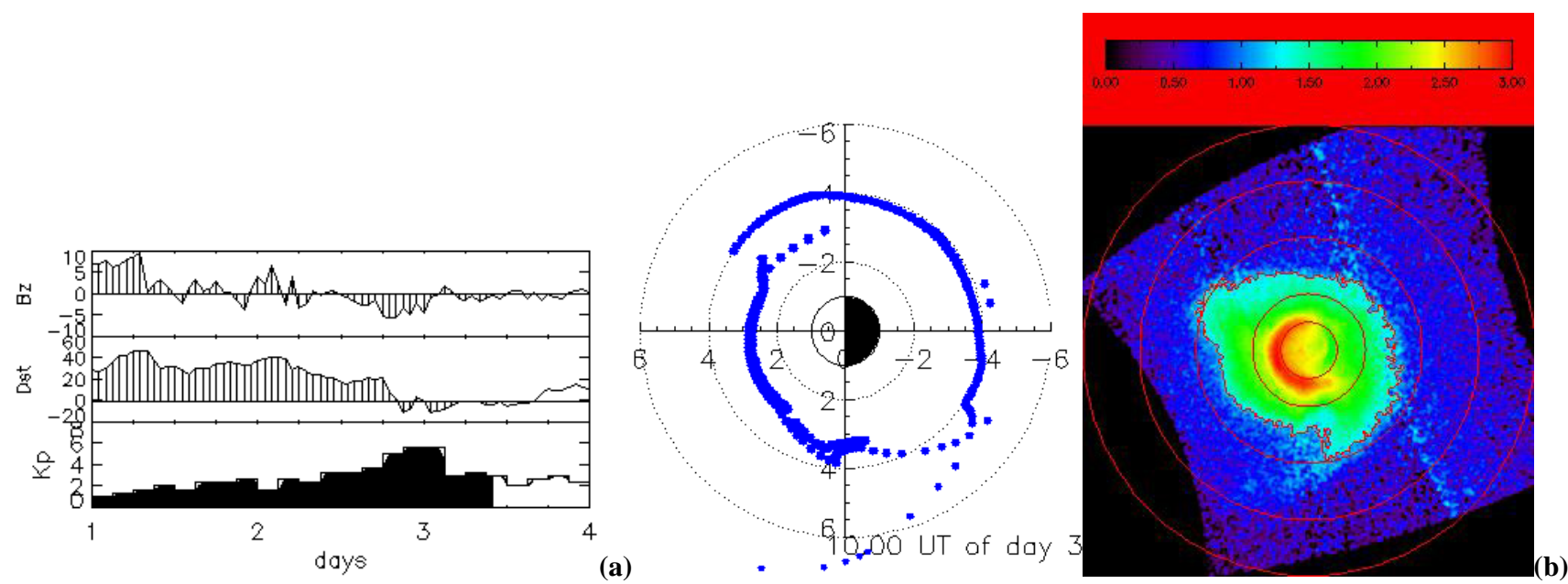

Fig. 6. Same as Fig. 1 for 10 June 2001, 10:00 ((a), simulation) and 09:57 UT ((b), EUV observation).

relative intensity threshold procedure provides slightly different plasmapause positions than those obtained by the manual procedure used by Spasojevic et al. (2003) to determine the plasmapause position.

Note that there is no free parameter (i.e. no ad hoc fetch fitting parameter) used in our simulations to improve the fit with observations. Only the observed values of $K_{p}$ are used as input with McIlwain's E5D and M2 electric and magnetic field models. Of course, it could be possible to improve the results of such model simulations by adding or adjusting some parameters, but the goal of the present study is not to obtain the "best fit" with the observations. The goal is to check whether the formation of the plasmapause by an interchange mechanism does approximately reproduce the overall feature and evolution of the plasmapause observed with the EUV instrument.

\subsection{June 2000}

Another example of plume formation was observed between 25 and 26 June 2000. Figure 8a shows the result of our simulation obtained at 21:00 UT on 25 June. The plasmapause is rather extended, due to the low values of $K_{p}$ in the previous hours. The plasmasphere is also quite circular due to the stationarity of $K_{p}$ during the previous $24 \mathrm{~h}$. The quiet period was, however, preceded by a substorm the day before, that is why the vestigial plasmapause is also shown closer to the Earth at $\mathrm{R}=3.8 R_{E}$ in the afternoon sector. The vestigial plasmapause corresponds to the inner points. Above this vestigial plasmapause, the outer flux tubes of the plasmasphere are supposed to refill up to the external plasmapause.

The EUV observations in Fig. 8b show the quasi-circular plasmasphere at 21:03 on 25 June. The observed plasmapause is quite close to the Earth and suggests that the outer flux tubes are not yet fully refilled. Note, nevertheless, that 


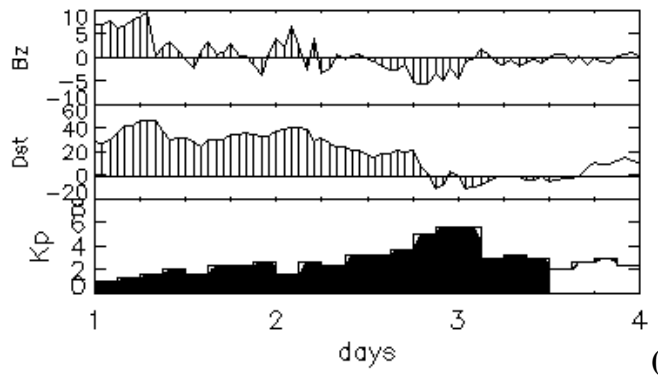

(a)
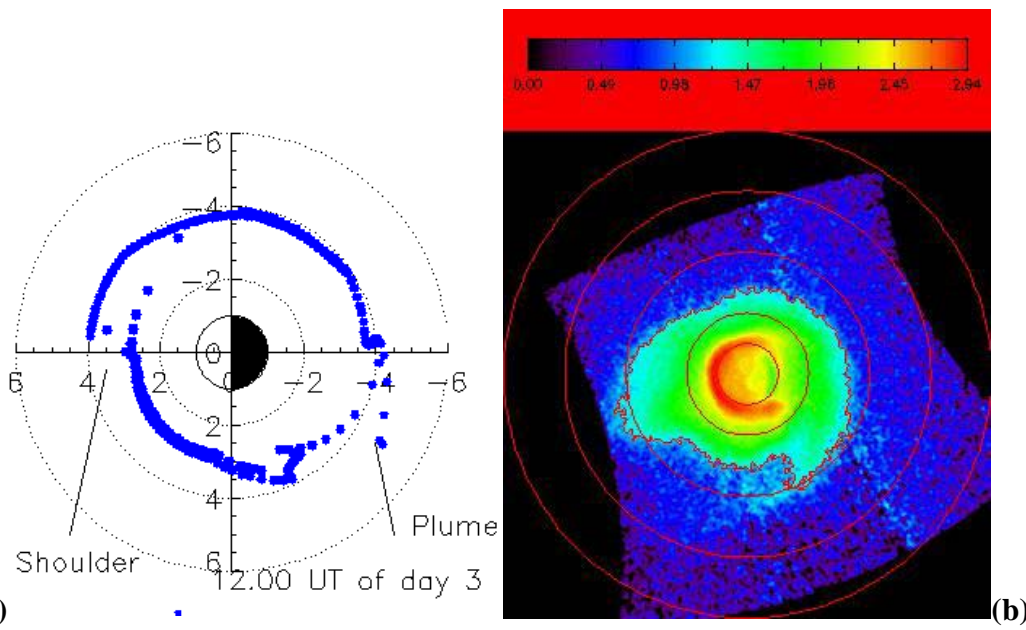

Fig. 7. Same as Fig. 1 for 10 June 2001, 12:00 ((a), simulation) and 12:00 UT ((b), EUV observation).
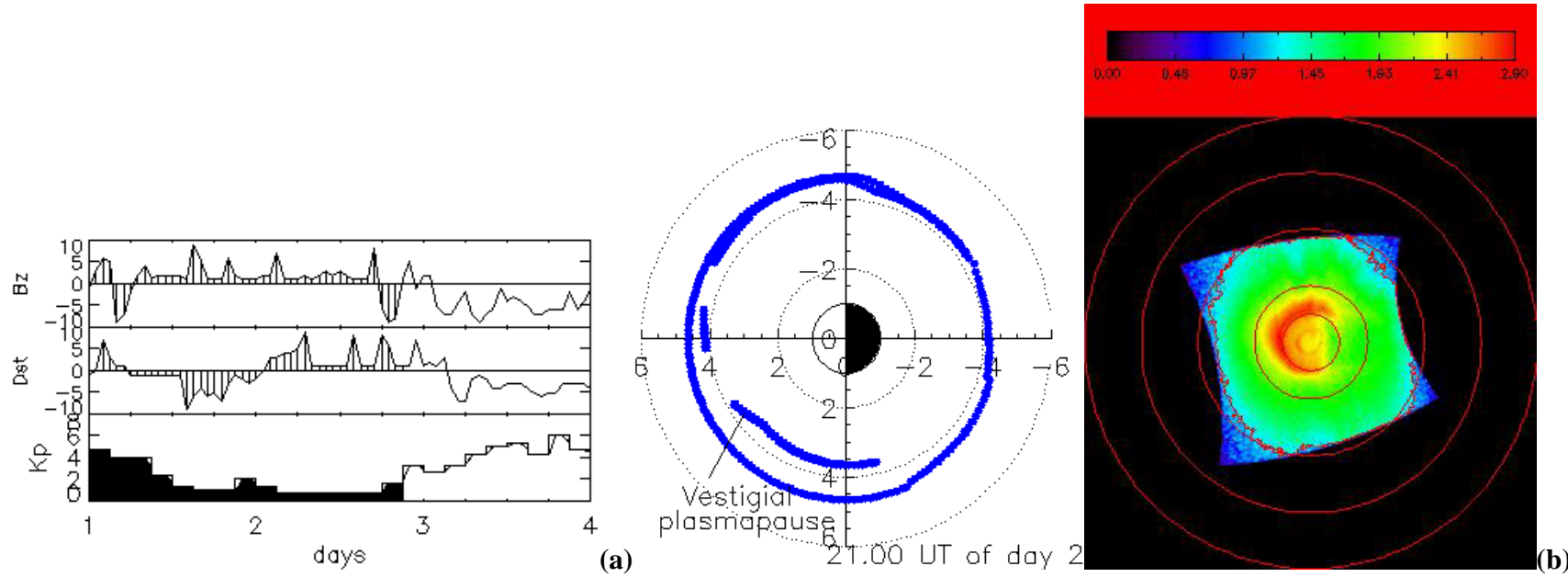

Fig. 8. Same as Fig. 1 for 25 June 2000, 21:00 UT ((a), simulation) and 21:03 UT ((b), EUV observation).

the satellite is located quite close to the Earth at that time, so that the global view of the plasmasphere is restricted.

In the dawn sector, one can see a density depletion that is mostly extended in the radial direction (a "channel"). These kinds of features were discovered in the EUV observations and permit one to measure the rotation velocity of the plasmasphere at different radial distances, which is often lower than co-rotation (Spasojevic et al., 2003). The observed channel can be due to a plume wrapping, as illustrated in the previous case of 10 June 2001, but other origins may be possible.

On 26 June $2000, K_{p}$ increases up to 6 during a geomagnetic substorm and this leads to the formation of a plume in our simulations. We show the development of the plume obtained in the simulation in Figs. 9a and b, at 03:00 and 09:00 UT. The formation of the plume begins in the pre-noon sector in the simulation, but it is only well formed and identifiable as a plume when it reaches the afternoon sector. Un- fortunately, EUV doesn't provide any observation during this period since the satellite IMAGE is too close to the Earth to give a global view of the plasmasphere.

In Fig. 10a, the result of the model is shown on 26 June 2000 at 16:00 UT. A nice plume has developed due to the $K_{p}$ increase and is now well structured. This plume is clearly seen in the same LT sector in the EUV image corresponding to the same time, shown in Fig. 10b. The position of the plasmapause is observed to be slightly closer to the Earth than in the simulation. The plasmapause density gradients are very sharp, as is often the case after such events, and the plasmapause position is well defined in the integrated intensity plots of EUV.

We have analyzed over ten of such events where $K_{p}$ increases. Although the results do not fit exactly the observations, our simulations reproduce rather satisfactorily the erosion of the plasmasphere followed by the formation of plumes during geomagnetic substorms. Plumes are always 

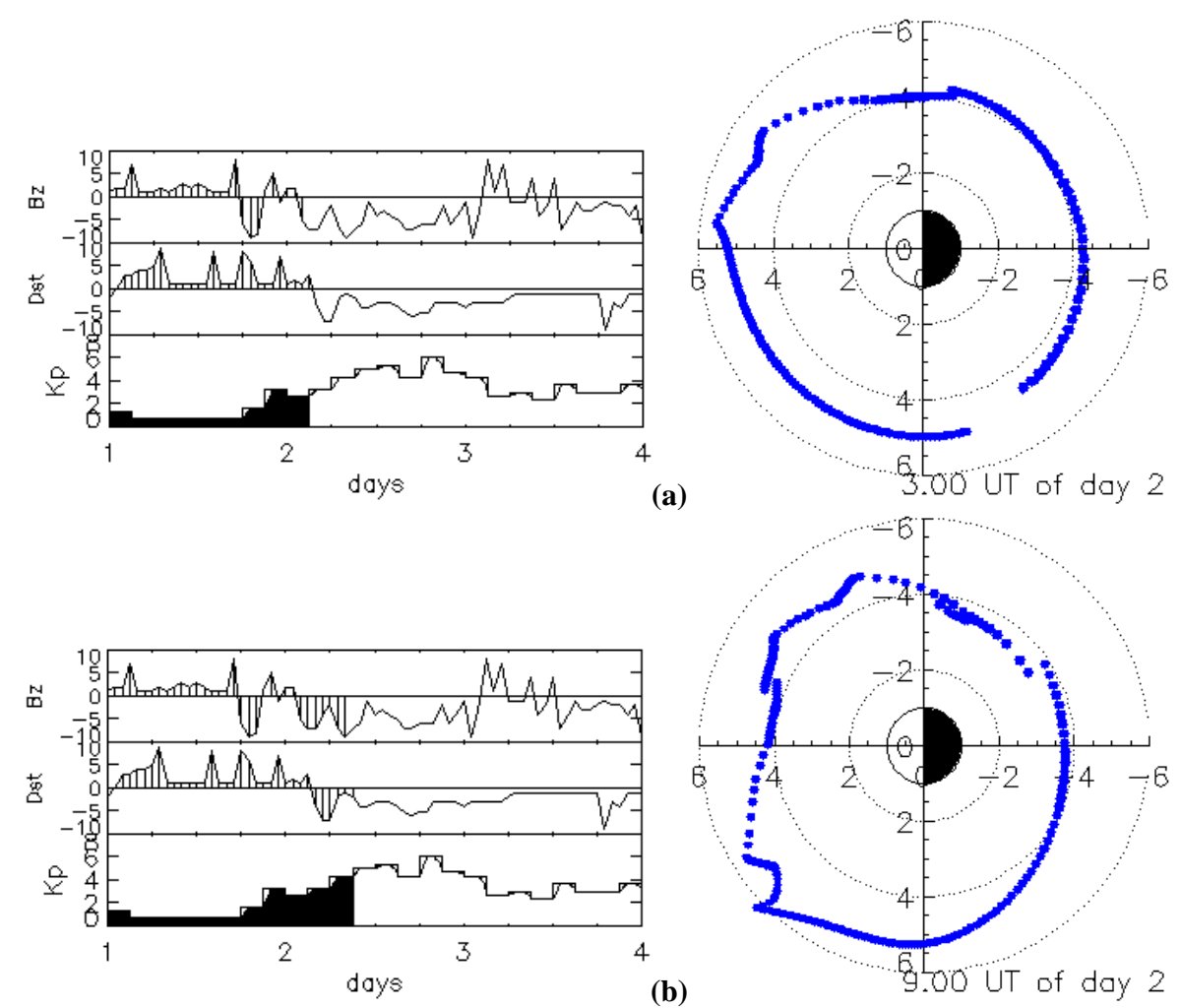

Fig. 9. Simulation of the development of the plume on 26 June 2000 at 03:00 UT (a) and 09:00 UT (b). IMAGE could not give global views of the plasmasphere during this period of time.

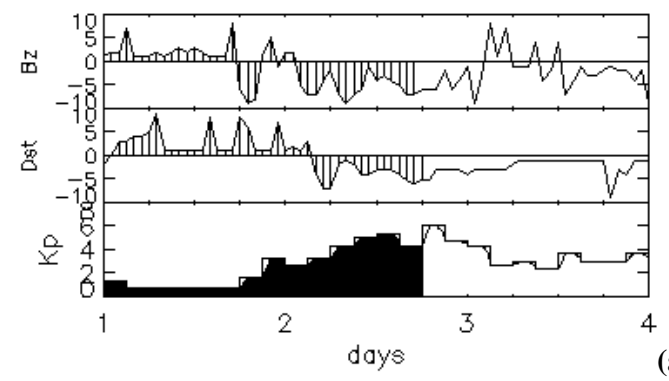

(a)

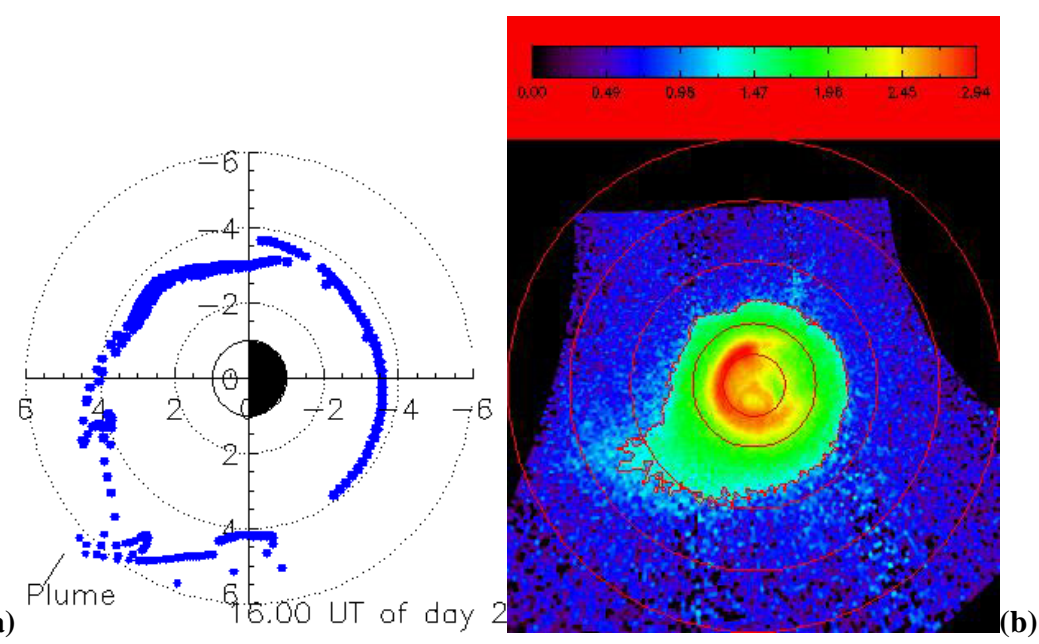

Fig. 10. Same as Fig. 1 for 26 June 2000, 16:00 UT ((a), simulation) and 15:56 UT ((b), EUV observation).

formed in the afternoon LT sector a few hours after an increase in $K_{p}$. Subsequently, the plumes slowly rotate when the geomagnetic activity decreases.

\section{During a geomagnetic storm: 24 May 2000}

During magnetic storms, $D_{s t}$ decreases during a main phase and then recovers over about one day. $K_{p}$ increases then usually up to high values ranging between 7 and 9 . For instance, on 24 May $2000, K_{p}$ increased up to 8 (see Fig. 11a, upper panel) and $D_{s t}$ drops to $-130 \mathrm{nT}$. Although the origin of geomagnetic storms and substorms might be different, the result 

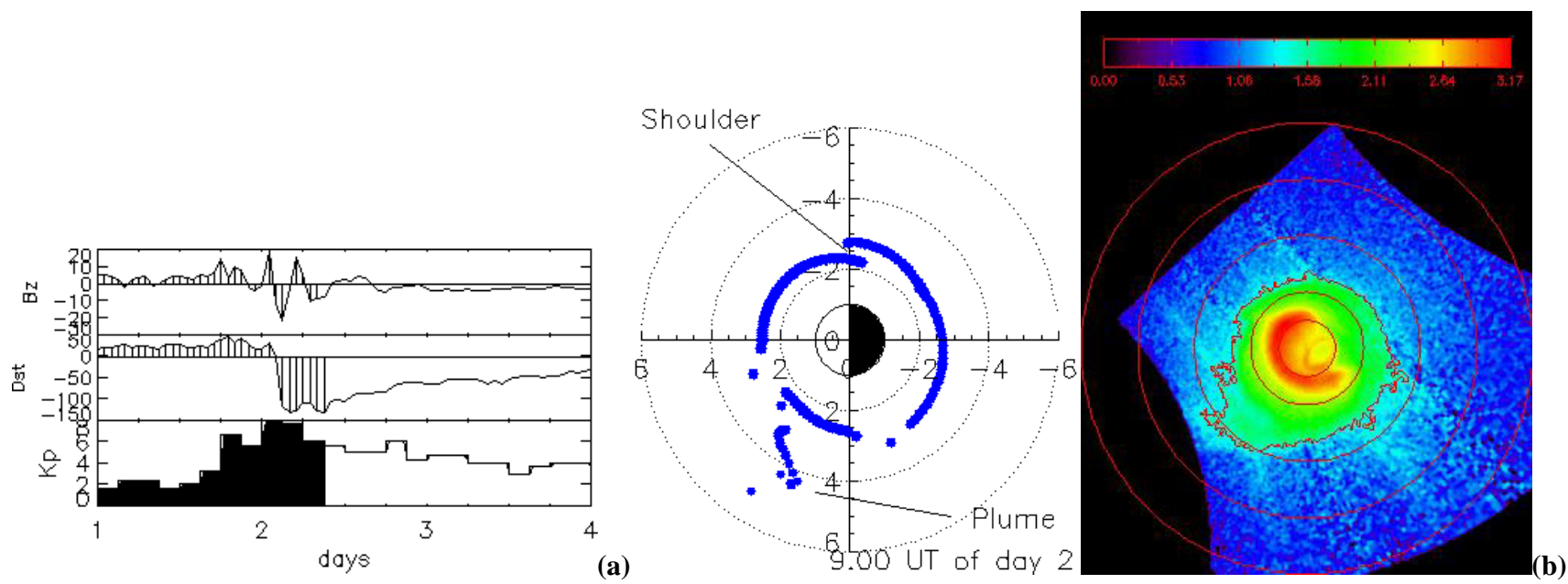

Fig. 11. Same as Fig. 1 for 24 May 2000, 09:00 ((a), simulation) and 09:02 UT ((b), EUV observation). A plume develops in the afternoon sector in the simulation as in the observations.
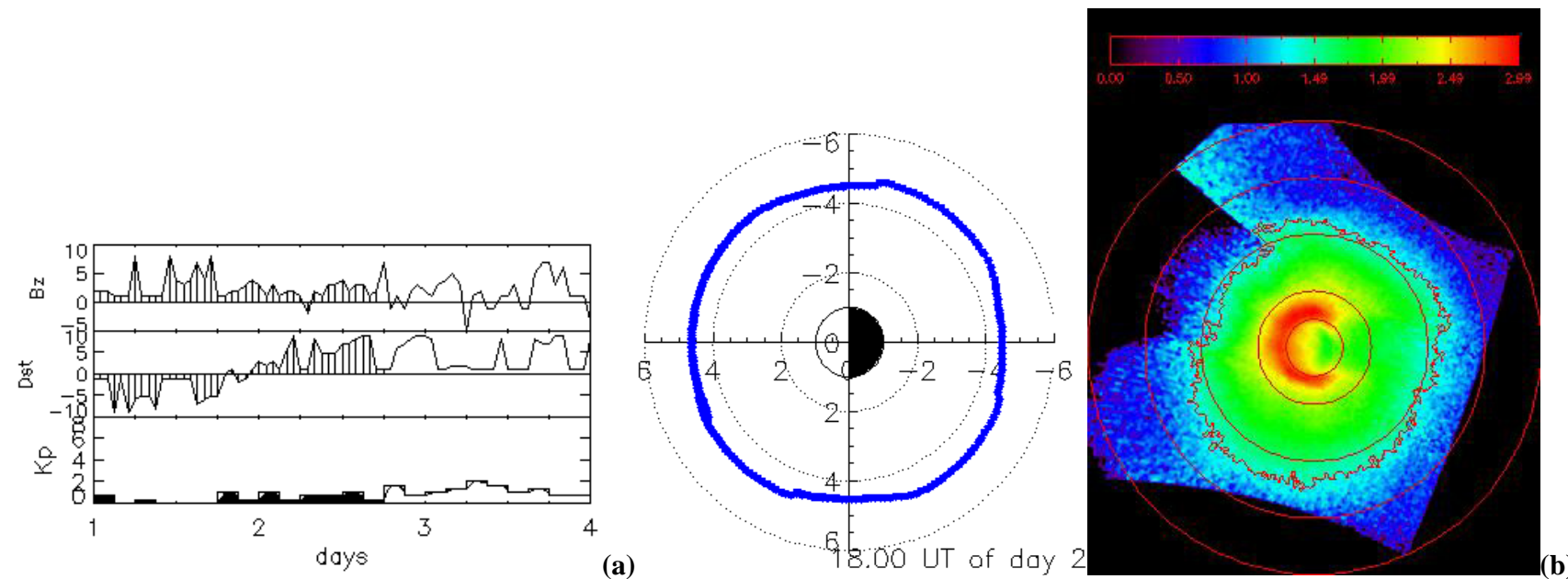

Fig. 12. Same as Fig. 1 for 2 May 2001, 18:00 ((a), simulation) and 17:58 UT ((b), EUV observation).

of the large increase in $K_{p}$ also has the effect to peel off the plasmasphere in the post-midnight region and to develop a well-defined plume.

Figure 11a shows the result of the simulation at 09:00 UT. A plume is formed due to the increase in $K_{p}$ and a shoulder due to the sharp decrease in $K_{p}$. Since the increase in $K_{p}$ is large, the plasma elements corresponding to the blue dots on Fig. 11a are spaced when the plume is formed. The plasmapause corresponds to an imaginary line joining these dots. The presence of two different boundaries in afternoon LT sector (one near Earth; one at higher radial distance) means that the plume which formed in the afternoon sector is, in fact, quite thin.

This figure can be compared with the observations, presented in Fig. 11b and also presented by Burch et al. (2001). The plume is well visible in the same LT sector. A shoulder is also present, although not so distinctly as in our simulations.
The radial distance of the plasmapause corresponds on average, to what is obtained in the simulation. It is only slightly closer to the Earth than during geomagnetic substorms.

\section{During a very quiet period: 2 May 2001}

When $K_{p}$ is very low during a long period of time, the plasmapause is located far from the Earth, since the flux tubes have been refilling for enough time to approach saturation. An example of a very quiet and extended period is presented in Fig. 12a by the result of the simulation at 18:30 UT on 2 May 2001, when $K_{p}$ remained lower than 2for over 2 days. The EUV observations at 18:28 UT are illustrated in Fig. 12b: the plasmasphere is very extended and the gradients in density are very smooth over the whole field of view. There does not appear to be a sharp knee in the plasma density profiles. Thus, the plasmapause position, as drawn 


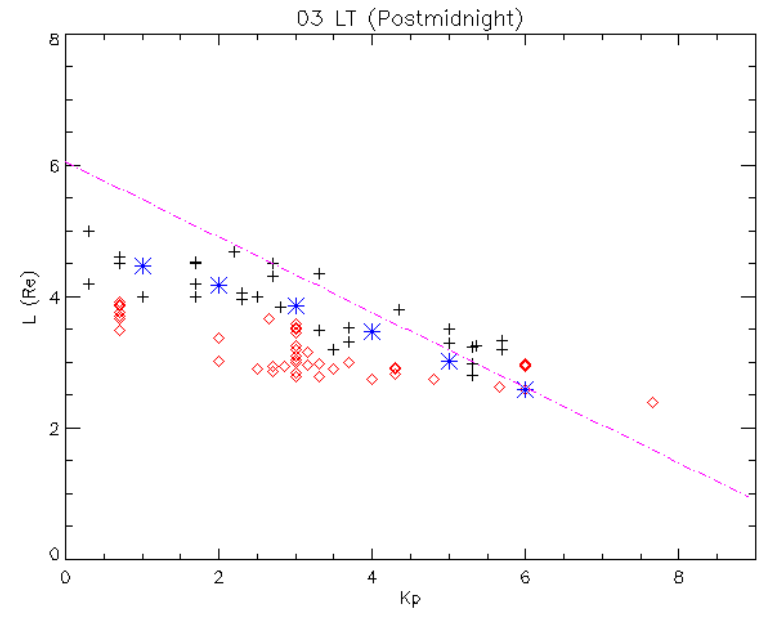

Fig. 13. Radial distance of the plasmapause observed at 03:00 MLT in the EUV observations for all 4 periods of time presented in this paper. When the $K_{p}$ index decreases, symbols are red diamonds $\diamond$; they are black crosses (+) when $K_{p}$ increases. The blue stars represent the plasmapause position found with the instability model for a constant value of $K_{p}$. The pink line corresponds to the linear relation found by Moldwin et al. (2002) between L and $K_{p}$ for average plasmapause crossings observed by CRRES in the dawn LT sector between August 1990 and October 1991.

by the red line, is very dependent on the adopted threshold value. In this figure, like in the other figures in this paper, the threshold is $40 \%$ of the maximum intensity.

Whistler and satellite observations have also shown that after a quiet period of time, the density gradients are generally very smooth. On the contrary, the plasmapause is very sharp after a peeling-off event, when the level of geomagnetic activity increases abruptly!

\section{Radial distance of the plasmapause}

It is also interesting to perform a statistical study of the radial distance of the plasmapause observed by EUV in the postmidnight sector, where the plasmapause is formed with the mechanism of plasma instability. The plasmapause positions at 03:00 LT are displayed in Fig. 13 as a function of $K_{p}$. The crosses and diamonds represent the radial distance of the plasmapause observed by EUV at 03:00 LT for the 4 periods of time considered in this paper.

Red diamonds are used when the $K_{p}$ index decreases (following a geomagnetic storm or substorm); black crosses are used when $K_{p}$ increases (following a quiet period). There is a significant scatter among the experimental results, indicating that the plasmapause also depends on other parameters than $K_{p}$, but it is quite clear that the plasmapause position is located closer to the Earth when $K_{p}$ is large. This confirms many earlier whistler and spacecraft observations. The pink straight line in Fig. 13 corresponds to the linear relation found by Moldwin et al. (2002) between $\mathrm{L}$ and $K_{p}$ for CRRES data in the dawn LT sector.
This figure also shows that the plasmapause is closer to the Earth when $K_{p}$ decreases (diamonds) than when $K_{p}$ increases (crosses), which indicates that the peeling-off effect takes place when geomagnetic activity is enhancing, and that during the following period of quieting, the plasmapause tends to expand radially outward. As a result of this followup radial expansion, the plasma density inside the plasmasphere is expected to be reduced, as indeed observed since many years through the whistler technique (Carpenter and Lemaire, 1997). The blue stars represent the theoretical plasmapause position predicted by the instability model in case $K_{p}$ would have been independent of time.

\section{Conclusions and discussion}

The following results are obtained from the direct comparison between the EUV observations from IMAGE and numerical simulations of the plasmapause position determined from the value of $K_{p}$ using the instability mechanism with the E5D electric field model:

1. Plumes are produced by our simulations after a moderate increase in $K_{p}$. They develop in the afternoon sector. The base of the plume rotates faster than its top during the quieting period following the enhanced magnetic activity, so that the plume is deformed and wraps in the dusk sector. Plumes are indeed observed by EUV during geomagnetic storms in the same LT sectors;

2. Observed shoulders are produced just at the end of magnetic substorms or storms; they generally develop one or two hours before a sharp decrease in $K_{p}$;

3. Very steep gradients of density are observed in the postmidnight sector, where the peeling-off effect is the most important, according to the instability scenario and the McIlwain E-field empirical model;

4. For a given value of $K_{p}$, the EUV data show that the plasmapause positions are rather widely scattered about an average value. The plasmapause position is not simply determined by the instantaneous $K_{p}$ value, but it also depends on its past history, i.e. if it was increasing or decreasing before the current time. On average, the plasmapause position is a nonlinear function of $K_{p}$, although a clear linear trend is found for large values of $K_{p}$;

5. The instability theory for the formation of the plasmapause qualitatively accounts for a range of dynamical plasmaspheric events and observed features;

6. The results of our simulations could be improved by introducing other effects or by adjusting some parameters in the simulations. For instance, in the present simulations, we use the empirical model of E5D that includes the co-rotation velocity and the convection velocity. Nevertheless, some studies have revealed that 
in the range $2<\mathrm{L}<4$, the plasma frequently rotates at a rate of $85-90 \%$ of the co-rotation (Sandel et al., 2003). The simulations adapted with a lower rotation velocity sometimes give a better agreement with the EUV observations. A plasmaspheric wind in the radial direction can also have some effects.

We present here some typical cases of comparisons between our simulations of the plasmapause formation and EUV observations. Other cases also show a rather good agreement. But of course, these results do not mean that all features observed with EUV can be simulated in detail with the numerical code. Moreover, these results do not exclude that tails and shoulders can also be formed by other proposed mechanisms. The role of the sub-auroral polarization stream (SAPS) in the plume formation and in the sunward transport of plasma from dusk is, for instance, more and more emphasized in the recent literature (Foster and Vo, 2002; Goldstein et al., 2003; Spasojevic et al., 2004).

It is also clear that the quasi-static E5D electric field model has inherent limitations and is certainly not the ultimate electric field we need for space weather predictions. E5D has a time resolution limited to $3 \mathrm{~h}$, due to its $K_{p}$ dependence. Moreover, Liemohn et al. (2004) noted that E5D is good at predicting the plasmapause location in the post-midnight sector, but it is too weak in the noon sector to account for the EUV observations during the recovery phase of the 17 April 2002 magnetic storm.

Despite these limitations, such comparisons and future additional ones allow us to identify the similarities and the discrepancies between the results of the simulations and the observations. This work helps to improve the theory for the formation of the plasmapause, as well as the empirical models of the inner magnetospheric electric field.

Acknowledgements. The authors thank B. Sandel, Lunar and Planetary Laboratory, University of Arizona, Tucson, USA and D. L. Gallagher, NASA, National Space Science and Technology Center, Huntsville, USA for the access to the EUV observations of the satellite IMAGE. The authors thank also J. Lemaire for discussions and commenting the manuscript. V. Pierrard thanks the Belgian PPS for the financial support (grant $\mathrm{MO} / 35 / 010$ ).

Topical Editor T. Pulkkinen thanks P. Décréau and another referee for their help in evaluating this paper.

\section{References}

Burch, J. L., Mende, S. B., Mitchell, D. G., Moore, T. E., Pollock, C. J., Reinisch, B. W., Sandel, B. R., Fuselier, S. A., Gallagher, D. L., Green J. L., Perez, J. D., and Reiff, P. H.: Views of Earth's Magnetosphere with the IMAGE Satellite, Science, 291, 619624, 2001

Carpenter, D. L. and Anderson, R. R.: An ISEE/whistler model of equatorial electron density in the magnetosphere, J. Geophys. Res., 97(A2), 1097-1108, 1992.

Carpenter, D. L. and Lemaire, J.: Erosion and recovery of the plasmasphere in the plasmapause region, Space Sci. Rev., 80, 153 179, 1997.
Chappell, C. R., Harris, K. K., and Sharp, G. W.: The dayside of the plasmasphere, J. Geophys. Res., 76, 7632-7647, 1971.

Dandouras, I., Pierrard, V., Goldstein, J., Vallat, C., Parks, G. K., Rème, H., Gouillart, C., Sevestre, F., McCarthy, M., Kistler, L. M., Klecker, B., Korth, A., Bavassano-Cattaneo, M. B., Escoubet, Ph., and Masson, A.: Multipoint observations of ionic structures in the Plasmasphere by CLUSTER-CIS and comparisons with IMAGE-EUV observations and with Model Simulations, accepted for publication in Yosemite 2004 AGU Monograph, 2004.

Darrouzet, F., Décréau, P. M. E., De Keyser, J., Masson, A., Gallagher, D. L., Santolik, O., Sandel, B. R., Trotignon, J. G., Rauch, J. L., Le Guirriec, E., Canu, P., Sedgemore, F., André, M., and Lemaire, J. F.: Density structures inside the plasmasphere: Cluster observations, Ann. Geophys., 22, 2577-2585, 2004,

SRef-ID: 1432-0576/ag/2004-22-2577.

Foster, J. C. and H. B. Vo: Average characteristics and activity dependence of the subauroral polarization stream, J. Geophys. Res., 107(A12), 1475, doi:10.1029/2002JA009409, 2002.

Goldstein, J., Sandel, B. R., Hairston, M. R., and Reiff, P. H.: Control of plasmaspheric dynamics by both convection and subauroral polarization stream, J. Geophys. Res., 30(24), 2243, doi:10.1029/2003GL018390, 2003.

Goldstein, J., Spiro, R. W., and Reiff, P. H., et al.: IMF-driven overshielding electric field and the origin of the plasmaspheric shoulder of 24 May 2000, Geophys. Res. Lett., 16, 66-1:4, doi:10.1029/2001GL014534, 2002.

Goldstein, J., Sandel, B. R., Thomsen M. F., Spasojevic, M., and Reiff, P. H.: Simultaneous remote sensing and in situ observations of plasmaspheric drainage plumes, J. Geophys. Res., 109(A03202), doi:10.1029/2003JA010281, 2004.

Grebowsky, J. M.: Model study of plasmapause motion, J. Geophys. Res., 75, 4329-4333, 1970.

Lemaire, J.: The "Roche limit" of ionospheric plasma and the formation of the plasmapause, Planet. Space Sci., 22, 757-766, 1974.

Lemaire, J. F.: Frontiers of the plasmasphere (Theoretical aspects), Université Catholique de Louvain, Faculté des Sciences, Editions Cabay, Louvain-La-Neuve, ISBN-2-87077-310-2, 1985.

Lemaire, J. F.: The formation plasmaspheric tails, Phys. Chem. Earth (C), 25, 1-2, 9, 2000.

Lemaire J. F. and Gringauz, K. I.: With contributions from Carpenter, D. L. and Bassolo, V. (Ed.): The Earth's plasmasphere, Cambridge University Press, Cambridge, 1998.

Liemohn M. W., Ridley, A. J., Gallagher, D. L., Ober, D. M., and Kozyra J. U.: Dependence of plasmaspheric morphology on the electric field description during the recovery phase of the 17 April 2002 magnetic storm, J. Geophys. Res., 109(A03209), doi:10.1029/2003JA010304, 2004.

McIlwain, C. E.: A $K_{p}$ dependent equatorial electric field model, The Physics of Thermal plasma in the magnetosphere, Adv. in Space Res., 6 (3), 187-197, 1986.

Moldwin, M. B., Downward, L., Rassoul, H. K., Amin, R., and Anderson, R. R.: A new model of the location of the plasmapause: CRRES results, J. Geophys. Res., 107, 1339:1-9, 2002.

Nishida, A.: Formation of plasmapause, or magnetsopheric plasma knee, by the combined action of magnetospheric convection and plasma escape from the tail, J. Geophys. Res., 71, 5669-5679, 1966.

Pierrard V. and Lemaire, J.: Development of shoulders and plumes in the frame of the interchange instability mechanism for plasmapause formation, Geophys. Res. Lett., 31, 5(L05809), 
doi:10.1029/2003GL018919, 2004.

Sandel B. R., Goldstein, J., Gallagher, D. L., and Spasojevic, M.: Extreme ultraviolet imager observations of the structure and dynamics of the plasmasphere, Space Sci. Rev., 109, 25-46, 2003.

Spasojevic, M., Goldstein, J., Carpenter, D. L., Inan, U. S., Moldwin, M. B., and Reinisch, B. W.: Global response of the plasmasphere to a geomagnetic disturbance, J. Geophys. Res., 108, 1340, doi:10.1029/2003JA009987, 2003.
Spasojevic M., Frey, H. U., Thomsen, M. F., Fuselier, S. A., Gary, S. P., Sandel, B. R., and Inan, U. S.: The link between a detached subauroral proton arc and a plasmaspheric plume, Geophys. Res. Lett., 31(L04803), doi:10.1029/2003GL018389, 2004.

Yoshikawa, I., Yamazaki, A., Shiomi, K., Yamashita, K., Takizawa, Y., and Nakamura, M.: Evolution of the outer plasmasphere during low geomagnetic activity observed by the EUV scanner onboard Planet-B, J. Geophys. Res., 105(A12), 27 777-22 789, 2000 . 